\title{
An updated checklist of the marine Decapoda of Ascension Island, central Atlantic Ocean
}

\author{
S. DE GRAVE ${ }^{1}$, A. ANKER ${ }^{2}$, P.C. DWORSCHAK ${ }^{3}$, P.F. CLARK ${ }^{4}$ AND P. WIRTZ ${ }^{5}$ \\ ${ }^{1}$ Oxford University Museum of Natural History, Oxford, UK, ${ }^{2}$ Tropical Marine Science Institute, National University of Singapore, \\ 18 Kent Ridge Road, Singapore 119227, Republic of Singapore, ${ }^{3}$ Naturhistorisches Museum Wien, Burgring 7, A-1010 Wien, Austria, \\ ${ }^{4}$ Department of Life Sciences, The Natural History Museum, London SW7 5BD, UK, ${ }^{5}$ Centro de Ciências do Mar, Universidade do \\ Algarve, P-8000-117 Faro, Portugal
}

\begin{abstract}
The decapod Crustacea from Ascension Island are reported upon on the basis of major expeditions undertaken during 2008 and 2012, including several minor additional collections made in other years. Two species, Gnathophyllum americanum and Corallianassa longiventris are new records for the island bringing the total known marine decapod fauna to 75 species, of which 11 are currently endemic to Ascension Island.
\end{abstract}

Keywords: Ascension Island, Crustacea, marine Decapoda, checklist

Submitted 11 May 2014; accepted 7 August 2014; first published online 23 September 2014

\section{INTRODUCTION}

Ascension Island is an isolated landmass in the central Atlantic Ocean, about $3000 \mathrm{~km}$ east of Brazil and $3000 \mathrm{~km}$ west of Africa, with the nearest neighbour being St Helena, about $1300 \mathrm{~km}$ to the south. The island has been visited by numerous early scientific expeditions, with the first decapod, Grapsus adscensionis (Osbeck, 1765) being recorded by Linnaeus (1754, as Cancer retusus Linnaeus, 1754). Over the ensuing decades, knowledge of the decapod fauna accumulated piecemeal, but was considerably enhanced by incidental collections sent to the late Ray Manning (United States National Museum, Smithsonian Institution, Washington DC) in 1970, which not only led to the description of two endemic taxa (see Chace \& Manning, 1972), but also resulted in two expeditions to the island by him and colleagues in 1971 and 1976. Accumulated knowledge of the decapod fauna of the island based on all collected material up to 1985 was summarized in the monograph of Manning \& Chace (1990). Since then, only two additional species have been recorded, Alpheus cedrici Anker \& De Grave, 2012 and Typton holthuisi De Grave, 2010 based on the 2008 Oxford University Museum of Natural History (OUMNH) expedition to the island. The present contribution reports on the other decapod species collected during the OUMNH 2008 expedition as well as further taxa collected during a 2012 expedition led by P. Brickle (Shallow Marine Surveys Group). Additionally, a number of specimens donated to OUMNH over the years are reported upon, as is a small, but significant, collection by P. Wirtz during 2014. Some of the material resulting from the 2008 expedition has already been reported upon (De Grave, 2010;

Corresponding author:

S. De Grave

Email: sammy.degrave@oum.ox.ac.uk
Anker \& De Grave, 2012) and is not further treated. All material examined is deposited in OUMNH, the Natural History Museum, London (NHMUK), Naturhistorisches Museum, Vienna (NHMW) or the Lee Kong Chian Natural History Museum (ex. Raffles Museum of Biodiversity Research), National University of Singapore (ZRC).

Much of the intertidal and subtidal coastline on Ascension is inaccessible, consequently the majority of collections were made at only a limited number of locations (Figure 1) with their geographical coordinates listed in Table 1.

Under each species treated, only the first record is listed for Ascension, fuller synonymies can be found in Manning \& Chace (1990). An update of the known marine decapod fauna of the island is provided (Table 2), extending the species list in Manning \& Chace (1990), with up to date nomenclature.

Size measurements: Size of carideans and stenopodideans was measured between the post-orbital margin and the posterior end of the carapace, along the dorsal line (pocl) for nonalpheids or including the rostrum (cl) for alpheids; brachyurans as carapace width (cw); paguroids as shield length (sl); callianassids as total length (tl) and carapace length ( $\mathrm{cl})$; and hippids as carapace length $(\mathrm{cl})$; all measurements being in $\mathrm{mm}$.

Abbreviations: female (Q); male $\left(\sigma^{7}\right)$; ovigerous (ov.); juvenile (juv.); individual (ind.); including (incl.) and field collection numbers $(\mathrm{f} \mathrm{cn})$, the latter only given when known.

\section{RESULTS}

Order DECAPODA Latreille, 1802 Infraorder STENOPODIDEA Spence Bate, 1888 Family STENOPODIDEA Claus, 1872 Odontozona anaphorae Manning \& Chace, 1990 (Figure 2A, B)

Manning \& Chace, 1990, p. 29; figures 14-15 


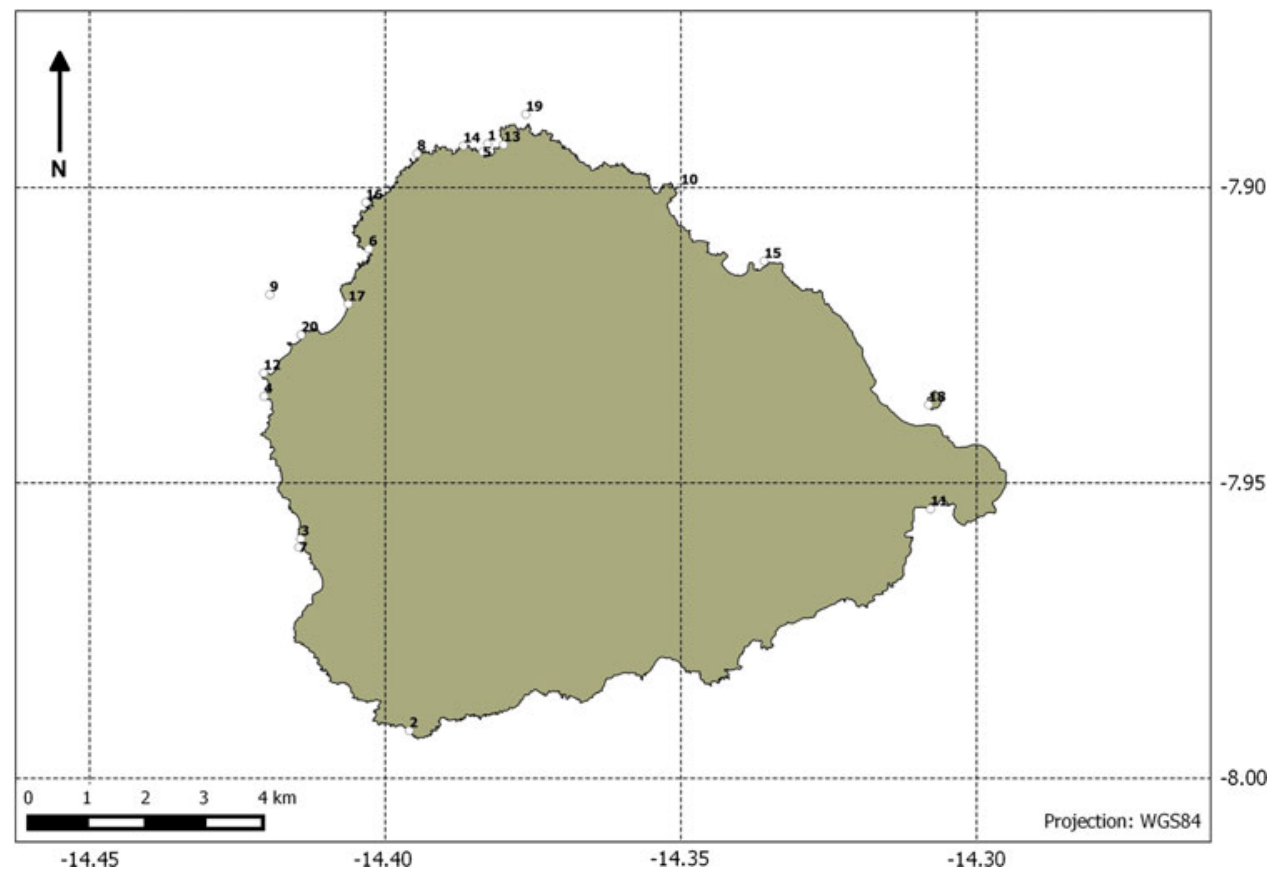

Fig. 1. Map of Ascension Island, showing sampling locations.

\section{MATERIAL EXAMINED}

fcn $\mathrm{C}_{41}$ /oo3, 2 ov. + , pocl, 4.6, 4.7, $1 \bigcirc^{7}$, pocl 4.5, western side of English Bay, in lava tube, $13 \mathrm{~m}, 4$ September 2012, OUMNH.ZC.2014.03.01/02/03.

\section{REMARKS}

This endemic species to Ascension was previously only known from the male holotype, collected in 1980 off North Point (Manning \& Chace, 1990). The present specimens demonstrate considerable variation in carapace and pereiopod spination, but are consistent with the diagnosis of the

Table 1. Geographic coordinates of sampling locations.

\begin{tabular}{llll}
\hline & Location name & Latitude & Longitude \\
\hline 1 & English Bay & -7.89257 & -14.38268 \\
2 & Shelly Beach & -7.99192 & -14.39593 \\
3 & Turtle Beach & -7.959463 & -14.414309 \\
4 & Collyer Point & -7.935336 & -14.420482 \\
5 & Wigan Pier & -7.893967 & -14.3835 \\
6 & Comfortless Cove & -7.910396 & -14.40278 \\
7 & McArthur Point & -7.960876 & -14.414595 \\
8 & Red Rock & -7.894233 & -14.3946 \\
9 & The Arches & -7.918117 & -14.419517 \\
10 & Porpoise Point & -7.9 & -14.35 \\
11 & South-East Bay & -7.954414 & -14.307776 \\
12 & Catherine Point & -7.931388 & -14.420561 \\
13 & Darby Beach & -7.89275 & -14.38 \\
14 & Two Hooks & -7.892917 & -14.3868 \\
15 & North East Bay & -7.91245 & -14.3359 \\
16 & Pyramid & -7.902517 & -14.403283 \\
17 & Long Beach & -7.919717 & -14.40625 \\
18 & Boatswain Bird Island & -7.936767 & -14.3081 \\
19 & Sudan Wreck & -7.8876 & -14.376217 \\
20 & Georgetown Pier & -7.924944 & -14.414223 \\
\hline
\end{tabular}

species. The previously unknown colour pattern is illustrated in Figure 2A, B.

Stenopus hispidus (Olivier, 1811)

Manning \& Chace, 1990, p. 31

\section{MATERIAL EXAMINED}

fcn ASC-009/010, 10, pocl 6.5, 19, pocl 7.3, west side of English Bay, hand collecting under ledge, $10 \mathrm{~m}, 10$ April 2008, OUMNH.ZC.2008.11.82/83.

Infraorder CARIDEA Dana, 1852

Family ALPHEIDAE Rafinesque, 1815

Alpheus bouvieri A. Milne Edwards, 1878

Manning \& Chace, 1990, p. 14.

\section{MATERIAL EXAMINED}

fcn ASC-013/014, 1 ov. + , pocl 10.0, 19 , pocl 4.7, east side of English Bay, in outfall stream of desalination plant, intertidal, 10 April 2008, OUMNH.ZC.2008.11.024/028; fcn ASC-068, 1 , pocl 3.6, English Bay, North Point, in rock pools, intertidal, 14 April 2008, OUMNH.ZC.2008.11.009; 2 juv., same location, 17 April 2008, OUMNH.ZC.2008.11.0008; fcn ASC-098, 7 ind., incl. ov. 9 , pocl 3.0-4.0, Shelly Beach, intertidal pools, 16 April 2008, OUMNH.ZC.2008.11.025/055; 1 , pocl 5.5, Turtle Beach Point, from algal clumps, intertidal, 11 April 2008, OUMNH.ZC.2008.11.054; 3 juv., Collyer Point, by blowhole, intertidal, 15 April 2008, OUMNH.ZC.2008.11.059.

$$
\text { Alpheus cedrici Anker \& De Grave, } 2012
$$

Anker \& De Grave, 2012, p. 2; figures 1-3.

MATERIAL EXAMINED

fcn $\mathrm{C}_{37} / 006,1$,, $\mathrm{cl}$ 6.0, Mars Bay, 15.1 m, 2 September 2012, OUMNH.ZC.2014.03.012; fcn $\mathrm{SP}_{3} / \mathrm{oO} 2,1$,, $\mathrm{cl} 10.3$, off Wigan 
Table 2. Species of marine Decapoda recorded from Ascension Island. The first record from the island is indicated, as well as their general biogeographic distribution.

\begin{tabular}{|c|c|c|c|}
\hline & Species & First record & Distribution \\
\hline \multicolumn{4}{|l|}{ Dendrobranchiata } \\
\hline Penaeidae & Metapenaeopsis gerardoi Pérez Farfante, 1971 & Manning \& Chace (1990) & Western and central Atlantic \\
\hline \multicolumn{4}{|c|}{ Nietapentaeopsts geraraot reted rantante, $19 / 1$} \\
\hline Spongicolidae & Microprosthema inornatum Manning \& Chace, 1990 & Manning \& Chace (1990) & Endemic \\
\hline \multirow[t]{2}{*}{ Stenopodidae } & Odontozona anaphorae Manning \& Chace, 1990 & Manning \& Chace (1990) & Endemic \\
\hline & Stenopus hispidus (Olivier, 1811) & Manning \& Chace (1990) & Pantropical-subtropical \\
\hline \multicolumn{4}{|l|}{ Caridea } \\
\hline \multirow{14}{*}{ Alpheidae } & Alpheus bouvieri A.Milne Edwards, 1878 & Manning \& Chace (1990) & Amphi-Atlantic, tropical \\
\hline & Alpheus cedrici Anker \& De Grave, 2012 & Anker \& De Grave (2012) & Endemic \\
\hline & Alpheus crockeri (Armstrong, 1941) & Manning \& Chace (1990) & Pantropical (?) \\
\hline & Alpheus dentipes Guérin, 1832 & Manning \& Chace (1990) & Central and eastern Atlantic \\
\hline & Alpheus holthuisi Ribeiro, 1964 & Manning \& Chace (1990) & Central and eastern Atlantic \\
\hline & Alpheus macrocheles (Hailstone, 1835) & Manning \& Chace (1990) & Central and eastern Atlantic \\
\hline & Alpheus paracrinitus Miers, 1881 & Ortmann (1893), as Alpheus ascensionis & Pantropical, species complex \\
\hline & Automate dolichognatha De Man, 1888 & Manning \& Chace (1990) & Pantropical, species complex \\
\hline & Metalpheus paragracilis (Coutière, 1897) & Manning \& Chace (1990) & Pantropical \\
\hline & Metalpheus rostratipes (Pocock, 1890) & Manning \& Chace (1990) & Pantropical \\
\hline & Parabetaeus hummelincki (Schmitt, 1936) & Manning \& Chace (1990); as Neoalpheopsis euryone & Amphi-Atlantic, tropical \\
\hline & Salmoneus setosus Manning \& Chace, 1990 & Manning \& Chace (1990) & Western and central Atlantic, tropical \\
\hline & Salmoneus teres Manning \& Chace, 1990 & Manning \& Chace (1990) & Western and central Atlantic, tropical \\
\hline & Synalpheus fritzmuelleri Coutière, 1909 & Manning \& Chace (1990) & Western and central Atlantic, tropical \\
\hline \multirow{2}{*}{ Gnathophyllidae } & Gnathophyllum americanum Guérin-Méneville, 1855 & Present contribution & Pantropical \\
\hline & Gnathophyllum ascensione Manning \& Chace, 1990 & Manning \& Chace (1990) & Endemic \\
\hline \multirow{4}{*}{ Hippolytidae } & Lysmata grabhami (Gordon, 1835) & Manning \& Chace (1990) & Amphi-Atlantic, tropical-subtropical \\
\hline & Lysmata moorei (Rathbun, 1901) & Chace (1972) & Amphi-Atlantic, tropical \\
\hline & Lysmata seticaudata (Risso, 1816) & Manning \& Chace (1990), as Lysmata intermedia & Central and eastern Atlantic \\
\hline & Thor manningi Chace, 1972 & Manning \& Chace (1990) & Western and central Atlantic \\
\hline \multirow[t]{4}{*}{ Palaemonidae } & Brachycarpus biunguiculatus (Lucas, 1846) & Manning \& Chace (1990) & Pantropical-subtropical \\
\hline & Pontonia pinnophylax (Otto, 1821) & Manning \& Chace (1990) & Central and eastern Atlantic \\
\hline & Typton ascensionis Manning \& Chace, 1990 & Manning \& Chace (1990) & Endemic \\
\hline & Typton holthuisi De Grave, 2010 & De Grave (2010) & Endemic \\
\hline Processidae & Processa packeri Manning \& Chace, 1990 & Manning \& Chace (1990) & Endemic \\
\hline Rhynchocinetidae & Rhynchocinetes rigens Gordon, 1936 & Manning \& Chace (1990) & Tropical-subtropical Atlantic \\
\hline \multicolumn{4}{|l|}{ Astacidea } \\
\hline Enoplometopidae & Enoplometopus antillensis Lütken, 1865 & Manning \& Chace (1990) & Amphi-Atlantic, tropical-subtropical \\
\hline \multicolumn{4}{|l|}{ Axiidea } \\
\hline Axiidae & Axiopsis serratifrons (A Milne-Edwards, 1873) & Manning \& Chace (1990) & Pantropical \\
\hline \multirow[t]{2}{*}{ Callianassidae } & Corallianassa hartmeyeri (Schmitt, 1935) & Manning \& Chace (1990) & Western and central Atlantic \\
\hline & Corallianassa longiventris (A. Milne-Edwards, 1870) & Present contribution & Western and central Atlantic \\
\hline \multicolumn{4}{|l|}{ Achelata } \\
\hline Palinuridae & Panulirus echinatus Smith, 1869 & Lenz \& Strunck (1914) & Amphi-Atlantic, tropical \\
\hline Scyllaridae & Scyllarides delfosi Holthuis, 1960 & Manning \& Chace (1990) & Western and central Atlantic \\
\hline Anomura & & & \\
\hline
\end{tabular}


Table 2. Continued

Diogenidae

Calcinus tubularis (Linnaeus, 1767)

Clibanarius rosewateri Manning \& Chace, 1990

Dardanus imperator (Miers, 1881)

Parapaguridae Sympagurus dimorphus (Studer, 1883)

Porcellanidae

Hippidae

Brachyura

Acidopsidae

Aethridae

Atelecyclidae

Calappidae

Cryptochiridae

Domeciidae

Dromiidae

Epialtidae

Grapsidae

Latreillidae

Panopeidae

Parthenopidae

Percnidae

Portunidae

Pseudoziidae

Raninidae

Xanthidae

Hippa testudinaria (Herbst, 1791)

Acidops cessaci (A. Milne-Edwards, 1878)

Osachila stimpsonii Studer, 1883

Atelecyclus rotundatus (Olivi, 1792)

Calappa galloides Stimpson, 1859

Mursia mcdowelli Manning \& Chace, 1990

Opecarcinus hypostegus (Shaw \& Hopkins, 1977)

Troglocarcinus corallicola Verrill, 1908

Domecia acanthophora (Desbonne, 1867)

Dromia erythropus (Edwards, 1771)

Dromia marmorea Forest, 1974

Dromia personata (Linnaeus, 1758)

Moreiradromia antillensis (Stimpson, 1859)

Acanthonyx sanctaehelena Chace, 1966

Grapsus adscensionis (Osbeck, 1765)

Pachygrapsus corrugatus (von Martens, 1872)

Pachygrapsus loveridgei Chace, 1966

Latreillia elegans Roux, 1830

Panopeus hartii Smith, 1869

Spinolambrus verrucosus (Studer, 1883)

Percnon abbreviatum (Dana, 1851)

Percnon gibbesi (H. Milne Edwards, 1853)

Plagusia depressa (Fabricius, 1775)

Laleonectes vocans (A. Milne-Edwards, 1878)

Portunus anceps (de Saussure, 1857)

Euryozius sanguineus (Linnaeus, 1771)

Ranilia constricta (A. Milne Edwards, 1880)
Manning \& Chace (1990)

Manning \& Chace (1990)

Ortmann (1893)

Stebbing (1914); as Eupagurus modicellus

Miers (1881); as Petrolisthes armatus

Apiomithrax violaceus (A. Milne-Edwards, 1867)

Cataleptodius olsoni Manning \& Chace, 1990

Microcassiope minor (Dana, 1852)

Nanocassiope melanodactylus (A. Milne-Edwards, 1868)

Paractaea rufopunctata africana Guinot, 1969

Platypodiella picta (A Milne-Edwards, 1869)

Williamstimpsonia denticulatus (White, 1848)
White (1847); as Remipes scutellatus

Manning \& Chace (1990)

Studer (1883)

Manning \& Chace (1990)

Manning \& Chace (1990)

Manning \& Chace (1990)

Kropp \& Manning (1987)

Kropp \& Manning (1987)

Manning \& Chace (1990)

Manning \& Chace (1990)

Manning \& Chace (1990)

Chace (1966); as Dromia erythropus

Manning \& Chace (1990); as Dromidia antillensi

Manning \& Chace (1990)

Manning \& Chace (1990)

Linnaeus (1754); as Cancer retusus

Manning \& Chace (1990)

Miers (1881); as Pachygrapsus transversus

Stebbing (1914)

Manning \& Chace (1990)

Studer (1883); as Lambrus verrucosus

Manning \& Chace (1990)

Miers (1881)

Manning \& Chace (1990)

Rathbun (1930)

Manning \& Chace (1990)

Linnaeus (1771)

Studer (1883), as Notopus atlanticus

Manning \& Chace (1990)

Manning \& Chace (1990)

Miers (1881)

Benedict (1893); as Actaea rufopunctata

Manning \& Chace (1990)

Manning \& Chace (1990); as Xanthodius denticulatus
Central and eastern Atlantic

Endemic

Central Atlantic

Central and southern Atlantic, deeper water

Amphi-Atlantic, tropical

Western and central Atlantic

Central and eastern Atlantic

Endemic

Central and eastern Atlantic

Amphi-Atlantic, tropical

Endemic

Western and central Atlantic

Amphi-Atlantic

Amphi-Atlantic

Western and central Atlantic

Central and eastern Atlantic

Central and eastern Atlantic

Western and central Atlantic

Central Atlantic

Amphi-Atlantic

Central and eastern Atlantic

Western and central Atlantic

Central Atlantic

Amphi-Atlantic

Western and central Atlantic

Endemic

Pantropical

Amphi-Atlantic, East Pacific

Amphi-Atlantic

Amphi-Atlantic, tropical

Western and central Atlantic

Central Atlantic

Amphi-Atlantic, tropica

Endemic

Amphi-Atlantic

Central and eastern Atlantic; eastern Pacific Central and eastern Atlantic, tropical

Central and eastern Atlantic, tropica

Amphi-Atlantic 


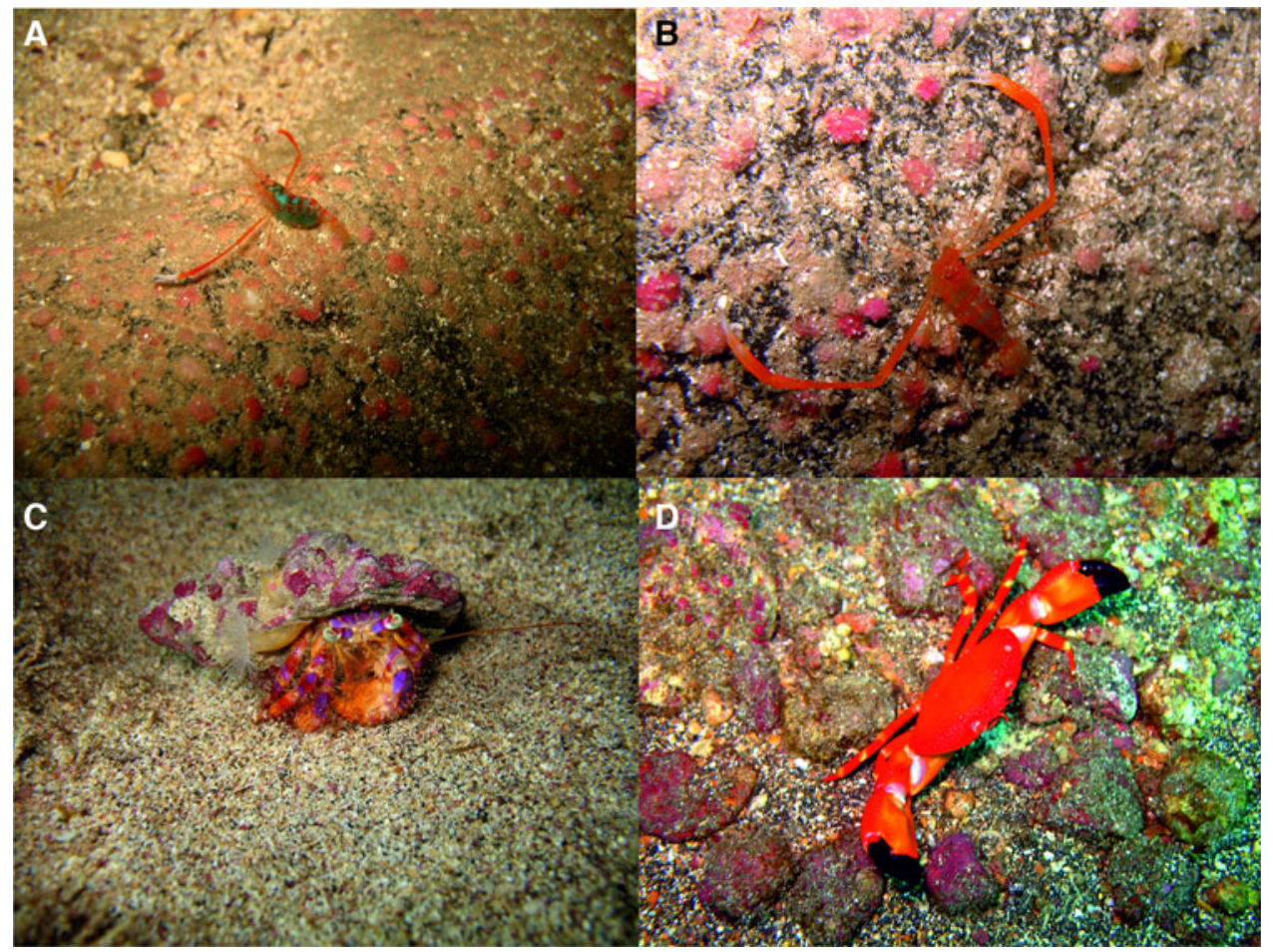

Fig. 2. Odontozona anaphorae Manning \& Chace, 1990: (A) female; (B) male. Dardanus imperator (Miers, 1881) (C). Euryozius sanguineus (Linnaeus, 1767) (D). All photos by P. Wirtz.

Pier, on settlement panel, $12.4 \mathrm{~m}, 4$ September 2012, OUMNH.2014.03.014.

\section{REMARKS}

This recently described species (Anker \& De Grave, 2012) was previously only known from the type series collected in English Bay. The additional records herein indicate the species to be more widespread around the island.

Alpheus crockeri (Armstrong, 1941)

Manning \& Chace, 1990, p. 14.

\section{MATERIAL EXAMINED}

fcn $\mathrm{C}_{45} / 001,1 \mathrm{O}^{7}$, pocl 5.8, 1 ov. + , pocl 5.2, Wigan Pier, $12.4 \mathrm{~m}$, 5 September 2012, OUMNH.ZC.2014.03.013; fcn $\mathrm{C}_{36} / 011,10^{7}$, pocl $6.8,2$, pocl $4.5,5.0$, Wigan Pier, 13.4 m, 2 September 2012, OUMNH.ZC.2014.03.016.

\section{REMARKS}

The present specimens conform to the description of the eastern Atlantic material reported under this name by Crosnier \& Forest (1966) and differ from the western Atlantic A. hortensis Wicksten \& McClure, 2003, in possessing a conspicuous dorso-distal tooth on the palm of the minor cheliped overhanging the base of the fingers, which is lacking in A. hortensis. Wicksten \& McClure (2003) did, however, express considerable doubt as to whether the eastern Atlantic populations reported under this name are identical to this widespread Indo-West Pacific species.

\section{Alpheus dentipes Guérin, 1832}

Manning \& Chace, 1990, p. 15.

\section{MATERIAL EXAMINED}

1 juv., Comfortless Cove, from Sabellaria reef, 1 m, 10 April 2008, OUMNH.ZC.2008.11.036; 4 ind., incl. ov. O, cl 2.3-5.4, same location, in coralline algal concretions, $2 \mathrm{~m}$, 13 April 2008, OUMNH.ZC.2008.11.015/029/037/046; 1 juv., same location, inside maerl balls, 2 m, 12 April 2008, OUMNH.ZC.2008.11.056; 3 우 cl 2.2-4.2, same location, underneath sponge, $5 \mathrm{~m}, 14$ April 2008, OUMNH.2008.11. 044; 10 ind., incl. ov. O, cl. 2.5-6.5, same location, from maerl ball, 5 m, 14 April 2008, OUMNH.ZC.2008.11.020; 12 ind., incl. ov. $O$, $\mathrm{cl} \mathrm{2.0-4.5,} \mathrm{McArthur} \mathrm{Point,} \mathrm{from} \mathrm{algal}$ clumps, intertidal, 12 April 2008, OUMNH.ZC.2008.11. 019/039/045/047; fcn ASC-122,1 9 , cl 4.8, east of McArthur Point, from coralline algae, intertidal pools, 17 April 2008, OUMNH.ZC.2008.11.038; fcn ASC-090, 2 juv., Collyer Point, by blowhole, intertidal, 15 April 2008, OUMNH.ZC. 2008.11.058; 1 juv., English Bay, north point, in intertidal rock pools, 14 April 2008, OUMNH.ZC.2008.11.021; fcn C10/005, 1 ov. O, cl 5.0, Red Rock, 23 August 2012, OUMNH.ZC.2014.03.017.

\section{Alpheus holthuisi Ribeiro, 1964}

Manning \& Chace, 1990, p. 15.

\section{MATERIAL EXAMINED}

fcn ASC-152, 1 ov. , , cl 5.0, western side of English Bay, under rocks, 15 m, 17 April 2008, OUMNH.ZC.2008.11.012; 10', cl 3.0, Collyer Point, by blowhole, intertidal, 15 April 2008, OUMNH.ZC.2008.11.060.

\section{Alpheus paracrinitus Miers, 1881}

Ortmann, 1893, p. 45 (as Alpheus ascensionis) Manning \& Chace, 1990, p. 16. 


\section{MATERIAL EXAMINED}

fcn ASC-032-043, 12 ind., cl 3.2-4.5, Comfortless Cove, in coralline algal concretions, $2 \mathrm{~m}, 13$ April 2008, OUMNH. ZC.2008.11.003/004/013/023/032/051; 1 juv., same location, from Sabellaria reef, $1 \mathrm{~m}, 10$ April 2008, OUMNH.ZC.2008. 11.033; $1 \mathrm{O}^{7}, \mathrm{cl} 3.2$, same location, inside maerl balls, $2 \mathrm{~m}$, 12 April 2008, OUMNH.ZC.2008.11.027; 1 ov. 9 , cl 6.3, western side of English Bay, under rocks, $10 \mathrm{~m}, 17$ April 2008, OUMNH.ZC.2008.11.050; fcn ASC-151,1 9 , cl 5.7, same location, under rocks, $15 \mathrm{~m}$, OUMNH.ZC.2008.11.049; $1 \sigma^{7}, \mathrm{cl} 4.0$, English Bay, North Point, in intertidal rock pools, 14 April 2008, OUMNH.ZC.2008.11.022.

\section{REMARKS}

This species was first recorded from Ascension Island by Ortmann (1893) as Alpheus ascensionis Ortmann, 1893. Holthuis (1951) placed A. ascensionis into the synonymy of A. paracrinitus, therein followed by Crosnier \& Forest (1966). Although a species complex is involved, it seems likely that the Ascension population belongs to $A$. paracrinitus sensu stricto in view of the proximity to the type locality (Senegal).

Automate dolichognatha De Man, 1888

Manning \& Chace, 1990, p. 16.

\section{MATERIAL EXAMINED}

fcn ASC-158,1 ov. 9, cl 4.2, western side of English Bay, under rocks, 10 m, 17 April 2008, OUMNH.ZC.2008.11.002/011; fcn ASC-136, 137,1 ov $ᄋ, \mathrm{cl}_{4.1}, 2$ ㅇ, cl 3.7, 4.7, same location, 16 April 2008, OUMNH.ZC.2008.11.001/010/030.

\section{REMARKS}

Although the material is herein assigned to A. dolichognatha, it is however acknowledged that the true status of the central and also western Atlantic populations must await a full revision of what is undoubtedly a pantropical species complex.

\section{Metalpheus paragracilis (Coutière, 1897)}

Manning \& Chace, 1990, p. 16.

\section{MATERIAL EXAMINED}

$10^{7}, \mathrm{cl} 2.7$, Comfortless Cove, underneath sponge, $5 \mathrm{~m}, 14$ April 2008, OUMNH.ZC.2008.11.043; fcn ASC-029, 10', cl 2.7, 1 ov. O, cl 4.5, McArthur Point, from intertidal algal clumps, 12 April 2008, OUMNH.ZC.2008.11.40/42; fcn ASC-139, 1 ov. O , cl 4.0, English Bay, North Point, from intertidal rockpool, 16 April 2008, OUMNH.ZC.2008.11.041; fcn ASC-148, 1 ov. 9 , cl 4.5, western side of English Bay, under rocks, $15 \mathrm{~m}, 17$ April 2008, OUMNH.ZC.2008.11.026; fcn C19/o04, 1 9 , cl 4.7, 1 juv., The Arches, 22 m, 25 August 2012, OUMNH.ZC.2014.03.015.

\section{Metalpheus rostratipes (Pocock, 1890)}

Manning \& Chace, 1990, p. 16.

\section{MATERIAL EXAMINED}

1 ov. O, cl 4.8, McArthur Point, from intertidal algal clumps, 12 April 2008, OUMNH.ZC.2008.11.048; fcn ASC-120, 128, 5 ind. incl. ov. + , $\mathrm{cl}$ 2.0-3.2, east of McArthur Point, from coralline algae wash, intertidal pool, 17 April 2008, OUMNH ZC.2008.11.005/006; fcn ASC-006, 1 ov. O, cl 4.0, Comfortless Cove, from Sabellaria clump, 1 m, 10 April 2008, OUMNH.
ZC.2008.11.034; 2 juv., Turtle Beach Point, from intertidal algal clumps, 11 April 2008, OUMNH.ZC.2008.11.052/053.

Parabetaeus hummelincki (Schmitt, 1936)

Manning \& Chace, 1990, p. 17 (as Neoalpheopsis euryone)

\section{MATERIAL EXAMINED}

1 , $\mathrm{cl} 5.5$, western side of English Bay, under rocks, $15 \mathrm{~m}, 17$ April 2008, OUMNH.ZC.2008.11.031.

\section{REMARKS}

This species was first recorded from Ascension Island by Manning \& Chace (1990) as Neoalpheopsis euryone, following Banner \& Banner (1985) who considered there to be only a single, pantropical species. Following the synonymization of Neoalpheopsis Banner, 1953 with Parabetaeus Coutière, 1896 in Nomura \& Anker (2001), the Atlantic species was tentatively resurrected (as P. hummelincki) by Anker (2007).

Salmoneus setosus Manning \& Chace, 1990

Manning \& Chace, 1990, p. 17

\section{MATERIAL EXAMINED}

fcn ASC-028, 1 ov. 9, cl 3.0, McArthur Point, from algal clumps, intertidal, 12 April 2008, OUMNH.ZC.2008.11.007; fcn ASC-053/056, 1 ov. + , cl 3.0, 1 juv., Comfortless Cove, in coralline algal concretions, $2 \mathrm{~m}, 13$ April 2008, OUMNH. ZC.2008.11.016/073.

\section{REMARKS}

Although originally described from Ascension Island, the species is now also known from Atol das Rocas and Fernando de Noronha, Brazil (Anker, 2007), as well as the Yucatan Peninsula, Mexico (Anker, 2010),

\section{Synalpheus fritzmuelleri Coutière, 1909}

Manning \& Chace, 1990, p. 22

\section{MATERIAL EXAMINED}

1 ov. O, cl 3.5, Comfortless Cove, in coralline algal concretions, 2 m, 13 April 2008, OUMNH.ZC.2008.11.014; 1 ov. 9 , cl 3.4, same location, from Sabellaria clumps, $1 \mathrm{~m}, 10$ April 2008, OUMNH.ZC.2008.11.035.

\section{Family GNATHOPHYLLIDAE Dana, 1852}

Gnathophyllum americanum Guérin-Méneville, 1855

\section{MATERIAL EXAMINED}

1 juv., pocl 1.2, English Bay, $12 \mathrm{~m}$, under stone, leg. P. Wirtz \& P. Nahke, 6 February 2014, OUMNH.ZC.2014.01.020.

\section{REMARKS}

A further individual of this distinctly coloured species was also observed by PW when turning over a stone in $13 \mathrm{~m}$ depth in English Bay on 10 September 2012. The animal escaped before it could be photographed or collected. These constitute the first record of this pantropical species for Ascension Island and the central Atlantic.

\section{Gnathophyllum ascensione Manning \& Chace, 1990}

Manning \& Chace, 1990, p. 11. 


\section{MATERIAL EXAMINED}

fcn ASC-119, 1 , , pocl 1.5, east of MacArthur Point, intertidal pool, under Diadema ascensionis, 17 April 2008, OUMNH. ZC.2008.11.075.

\section{REMARKS}

The specimen had a uniform black colour. The type series was found associated with Echinometra lucunter polypora, the present specimen constituting a new host record.

\section{Family HIPPOLYTIDAE Spence Bate, 1888 Lysmata grabhami (Gordon, 1935)}

Manning \& Chace, 1990, p. 23.

\section{MATERIAL EXAMINED}

2 ind., pocl 8.0, 9.4, Porpoise Point, under rocks, $14 \mathrm{~m}$, 19 June 2010, leg. RAF diving team, OUMNH.ZC.2010.04. 028/029; fcn C32/004, 2 ind., pocl 10.0-11.0, Stack in South-East Bay, $25 \mathrm{~m}, 1$ September 2012, OUMNH.ZC. 2014.03.018.

\section{Lysmata moorei (Rathbun, 1901)}

Chace, 1972, p. 128; Manning \& Chace, 1990, p. 23

\section{MATERIAL EXAMINED}

fcn ASC-96-111, 25 ind., pocl 2.1-3.5, Shelly Beach, in intertidal pools, 16 April 2008, OUMNH.ZC.2008.11.063/064/065/ 066/096/097/098/o99/100; 1 ind., pocl 3.9, Red Rock, 15 m, from crack in rock, leg. P. Wirtz \& P. Nahke, 9 February 2014, OUMNH.ZC.2014.01.021.

\section{Thor manningi Chace, 1972}

Manning \& Chace, 1990, p. 24

\section{MATERIAL EXAMINED}

fcn Q27/o03, 1 ov. O, pocl 1.5, Comfortless Cove, $10 \mathrm{~m}, 28$ August 2012, OUMNH.ZC.2014.03.010; 1 ov. + , pocl 1.7, 1 ind., English Bay, $10 \mathrm{~m}$, in crack in rock, leg. P. Wirtz \& P. Nahke, 9 February 2014, OUMNH.ZC.2014.01.018.

\section{REMARKS}

One of the individuals is noteworthy by possessing an acute branchiostegal tooth on the right-hand side of the carapace, with all other morphological features, as well as the colour pattern (photo by PW), fitting the diagnosis of T. manningi.

\section{Family PALAEMONIDAE Rafinesque, 1815 Brachycarpus biunguiculatus (Lucas, 1846)}

Manning \& Chace, 1990, p. 9.

\section{MATERIAL EXAMINED}

fcn ASC-116, $10^{7}$, pocl 4.5, western side of English Bay, under rocks, 5-10 m, 16 April 2008, OUMNH.ZC.2008.11.076; fcn ASC-147, $1 \sigma^{7}$, pocl 6.2, same location, under rocks, 15 m, 17 April 2008, OUMNH.ZC.2008.11.085; fcn C32/oo6, 1 , pocl 4.7 , Stack in South-East Bay, $25 \mathrm{~m}, 1$ September 2012, OUMNH.ZC.2014.03.05; fcn C36/oo9, $10^{7}$, pocl 3.5, 1 , pocl 4.4, Wigan Pier, $13.4 \mathrm{~m}, 2$ September 2012, OUMNH.ZC.2014.03.09; fcn C45/012, 1 ind., Wigan Pier, $12.4 \mathrm{~m}, 5$ September 2012, OUMNH.ZC.2014.03.07; 10', bopyrised, pocl 4.5, Red Rock, $15 \mathrm{~m}$, from crack in rock, leg. P. Wirtz \& P. Nahke, 9 February 2014, OUMNH.ZC. 2014.01.019.

Pontonia pinnophylax (Otto, 1821)

Manning \& Chace, 1990, p. 10.

\section{MATERIAL EXAMINED}

fcn ASC-011-012, 20', pocl 6.8, 7.8, 1 ov. O, pocl 8.5, W side of English Bay, from Pinna rudis, $10 \mathrm{~m}, 10$ April 2008, OUMNH.ZC.2008.11.078/081; fcn ASC-142-143, $10^{7}$, pocl 9.0, 1 , , pocl 11.2, same location, from Pinna rudis, $15 \mathrm{~m}$, OUMNH.ZC.2008.11.079/080; fcn C2/o03, 19, pocl 10.5, Wigan Pier, 12 m, 19 August 2012, OUMNH.ZC.2014.03.04; fcn C2/oo8, $10^{7}$, pocl 9.1, Wigan Pier, $12 \mathrm{~m}, 19$ August 2012, OUMNH.ZC.2014.03.06.

Family PROCESSIDAE Ortmann, 1896

Processa packeri Manning \& Chace, 1990

Manning \& Chace, 1990, p. 24.

\section{MATERIAL EXAMINED}

fon ASC-037-038, 13 ind., incl ov. 9 , pocl 1.9-3.2, Comfortless Cove, inside maerl balls, $2 \mathrm{~m}, 12$ April 2008, OUMNH.ZC.2008.11.057/069/089; $10^{7}$, pocl 1.6, 1 ov. + , pocl 1.1, same location, inside maerl balls, $5 \mathrm{~m}, 14$ April 2008, OUMNH.ZC.2008.11.070/088; 3 ind., pocl 1.0-1.1, same location, in coralline algal concretions, $2 \mathrm{~m}, 13$ April 2008, OUMNH.ZC.2008.11.090.

\section{REMARKS}

This species is the smallest processid shrimp worldwide, with females being ovigerous at $1.0-1.1 \mathrm{~mm}$ pocl.

\section{Family RHYNCHOCINETIDAE Ortmann, 1890 \\ Cinetorhynchus rigens (Gordon, 1936)}

Manning \& Chace, 1990, p. 9 (as Rhynchocinetes rigens)

\section{MATERIAL EXAMINED}

fcn C41/o02, 1 일 pocl 5.4, western side of English Bay, in lava tube, 13 m, 4 September 2012, OUMNH.ZC.2014.03.08.

Infraorder AXIIDEA de Saint Laurent, 1979 Family AXIIDAE Huxley, 1879

Axiopsis serratifrons (A. Milne-Edwards, 1873)

Manning \& Chace, 1990, p. 31; figures 16-17.

\section{MATERIAL EXAMINED}

1 juv., western side of English Bay, under rocks, 15 m, 17 April 2008, OUMNH.ZC.2008.11.093.

Family CALLIANASSIDAE Dana, 1852 Corallianassa longiventris (A. Milne-Edwards, 1870)

\section{MATERIAL EXAMINED}

fcn C45/024, 19, tl 71 cl 16.4, Wigan Pier, $12.4 \mathrm{~m}, 5$ September 2012, NHMW 25601; fcn C50/004, 1 ind. (incomplete, cephalothorax posterior to $\mathrm{P}_{3}$, pleon missing), same location, 12 m, 7 September 2012, NHMW 25602.

\section{REMARKS}

Differences between C. longiventris and C. hartmeyeri have been outlined by Schmitt (1935) and Manning (1988). The present specimens show all characters typical for 
C. longiventris. While Sakai (1999, 2005) considered both species valid (as Glypturus), Sakai (2011) synonymized hartmeyeri with longiventris without presenting any arguments for his action. Recent examination of many specimens from Florida, the Gulf of Mexico and Yucatan, however, have shown that both species differ from each other by several characters (D.L. Felder, personal communication, April 2014). The species is herein newly recorded for Ascension Island.

Infraorder ASTACIDEA Latreille, 1802

Family ENOPLOMETOPIDAE de Saint Laurent, 1988 Enoplometopus antillensis Lütken, 1865

Manning \& Chace, 1990, p. 31.

MATERIAL EXAMINED

1ㅇ, cl 20.5, Porpoise Point, under rocks, 14 m, 09/2010, leg. RAF diving team, OUMNH.ZC.2010.04.030.

REMARKS

A moult of this species was also found at English Bay on 9 September 2012, but not deposited.

\section{Infraorder ANOMURA MacLeay, 1838 Family DIOGENIDAE Ortmann, 1892 Dardanus imperator (Miers, 1881)} (Figure $2 \mathrm{C}$ )

Ortmann, 1893, p. 52.

\section{MATERIAL EXAMINED}

fcn C29/003, 1 ind., sl 4.8, Catherine Point, $10 \mathrm{~m}, 30$ August 2012, OUMNH.ZC.2014.03.019; fon Q27/oo4, 2. ind., Comfortless Cove, 10 m, 28 August 2012, OUMNH.ZC.2014 03.020; fcn C10/o02, 1 ind., sl 8.o, Red Rock, 23 August 2012, OUMNH.ZC.2014.03.021.

Family HIPPIDAE Latreille, 1825

Hippa testudinaria (Herbst, 1791)

Manning \& Chace, 1990, p. 41, Fig. 23 a.

\section{MATERIAL EXAMINED}

fen ASC 017, 4O', cl 10.4-13.3, $1913.4,1$ ov. 915.7 , hand collecting, surf zone, Turtle Beach, 11 April 2008, OUMNH. ZC.2008.11.0094; fcn IT7/oo1, 4 ov. O, cl 13.5-14.1, English Bay beach, 22 August 2012, NHMUK 2014.838-840.

Infraorder BRACHYURA Linnaeus, 1758 Family ACIDOPSIDAE Števčić, 2005

Acidops cessaci (A. Milne Edwards, 1878)

Manning \& Chace, 1990, p. 52.

\section{MATERIAL EXAMINED}

fcn ASC-155, 1 ㅇ, cw 17.3, 15 m, west side of English Bay, 17 April 2008, NHMUK 2014.561; fcn C11/008, 1 , cw 16.2, $9 \mathrm{~m}$, Darby Wreck arch, 23 August 2012, NHMUK 2014.841; fcn C18/004, $1 \mathrm{O}^{7}$, cw 12.9, 8.7 m, Darby Beach, 25 August 2012, not registered; fcn $\mathrm{C}_{50} / 005,1$ ov. $\mathrm{O}$, cw 16.4, 12 m, Wigan Pier, 7 September 2012, NHMUK 2014.842

\section{Family CALAPPIDAE De Haan, 1833}

Calappa galloides Stimpson, 1859
MATERIAL EXAMINED

fcn C50/007, $1 \sigma^{7}$, cw 17.6, 12 m, Wigan Pier, 7 September 2012, not registered.

Family CRYPTOCHIRIDAE Paulson, 1875

Troglocarcinus corallicola Verrill, 1908

Manning \& Chace, 1990, p. 69.

\section{MATERIAL EXAMINED}

fcn ASC-045, $10^{7}, \mathrm{cw} 1.8,2$ ov. + , cw 3.5, $2 \mathrm{~m}$, Comfortless Cove, 13 April 2008, NHMUK 2014.562-564.

Family EPIALTIDAE MacLeay, 1838

Acanthonyx sanctaehelenae (Chace, 1966)

Manning \& Chace, 1990, p. 47.

\section{MATERIAL EXAMINED}

fcn ASC-008, 10', cw 2.3, from Sabellaria Reef, $1 \mathrm{~m}$, Comfortless Cove, 10 April 2008, NHMUK 2014.759; fcn ASC-033, 1 juv., $\mathrm{cw}$ 2.0, hand collecting, intertidal, from algal clumps, McArthur Point, 12 April 2008, NHMUK 2014.562-564; fcn ASC-066, 19, cw 2.0, intertidal, in rock pools, English Bay, North Point, 14 April 2008, NHMUK 2014.770; fcn ASC-123, $10^{7}, \mathrm{cw}$ 2.9, from coralline algae, intertidal pools, east of McArthur Point/Turtle Beach, 17 April 2008, NHMUK 2014.771.

Apiomithrax violacens (A. Milne-Edwards, 1868)

Manning \& Chace, 1990, p. 47.

\section{MATERIAL EXAMINED}

fcn C25/o07, $10^{7}$, cW 23.2, Comfortless Cove, 29 August 2012, not registered; fen $\mathrm{C}_{4} 6$ 003, $10^{7}$, cw 8.7, English Bay, 5 September 2012, NHMUK 2014.843; fcn C55 002, 1 juv., 6.5. Two Hooks to English Bay, 9 September 2012, NHMUK 2014.844; fcn ASC-156 in rock pools, intertidal English Bay, North Point, 17 April 2008, OUMNH 2008-11-0077.

\section{Family GRAPSIDAE MacLeay, 1838} Grapsus adscensionis (Osbeck, 1765)

Manning \& Chace, 1990, p. 63.

\section{MATERIAL EXAMINED}

fcn ASC-070, 3 juv., cw 7.2-9.1, on rocks by blowhole, intertidal, Collyer Point, 13 April 2008, NHMUK 2014.562-564; fcn ASC-071, 2 juv., cw 8.9, 11.8, on rocks by blowhole, intertidal, Collyer Point, 13 April 2008, NHMUK 2014.568-569; fcn ASC-076, 1 juv., diving, from maerl ball, $5 \mathrm{~m}$, Comfortless Cove, 14 April 2008, NHMUK 2014.570; fcn ASC-084, 1 juv., cw 13.3, intertidal, Collyer Point, by blowhole, 15 April 2008, NHMUK 2014.571; fcn ASC-087, 1 juv., $\mathrm{Cw}$ 9.5, intertidal, Collyer Point, by blowhole, 15 April 2008, NHMUK 2014.572; fcn ASC-089, 2 juv., cw 18.8, 20.8, intertidal, Collyer Point, by blowhole, 15 April 2008, NHMUK 2014.573-574; fcn ASC-118, 1 juv., cw 11.2, intertidal pools, east of McArthur Point/Turtle Beach, 17 April 2008, NHMUK 2014.575; 1 ov. $O$, cw 55, 1 , cw 58, $50^{7}$, cw 44-64, in outlet of desalination plant, English Bay, intertidal, 10 April 2008, OUMNH.ZC.2008-11-061.

\section{Pachygrapsus loveridgei Chace, 1966}

Manning \& Chace, 1990, p. 66. 


\section{MATERIAL EXAMINED}

fcn ASC-003, $10^{7}, \mathrm{cw} 4.2,1$ ov. ㅇ, cw 4.7, from Sabellaria Reef, $1 \mathrm{~m}$, Comfortless Cove, 10 April 2008, NHMUK 2014.576-577; fcn ASC-007, $10^{7}, \mathrm{cW} 4.3,1$ ov. ㅇ, cw 4.8, from Sabellaria reef, $1 \mathrm{~m}$, Comfortless Cove, 10 April 2008, NHMUK 2014.578-579; fcn ASC-018, $10^{7}$, cw 9.6, hand collecting, intertidal, from Sabellaria reef, Turtle Beach Point, 11 April 2008, NHMUK 2014.580; fcn ASC-019, 10', cw 5.8, 1 ov. , $\mathrm{cw}$ 8.9, hand collecting, intertidal, between oysters, Turtle Beach Point, 11 April 2008, NHMUK 2014.581-582; fcn ASC-036, 1 \% , cw 6.0, hand collecting, intertidal, from algal clumps, McArthur Point, 12 April 2008, NHMUK 2014.583-584; fcn ASC-040, 1 ㅇ, cw 4.7, inside maerl balls, $2 \mathrm{~m}$, Comfortless Cove, 13 April 2008, NHMUK 2014.585; fcn ASC-081, 9 $\sigma^{7}, \mathrm{cW} 4.3-5.7,3$ ㅇ, cW 4.0-4.5, intertidal, by blowhole, Collyer Point, 15 April 2008, NHMUK 2014.586-595; fcn ASC-082, 100', cW 3.6-7.3, 3 ㅇ, cw 6.0-4.1, intertidal, by blowhole, Collyer Point, 15 April 2008, NHMUK 2014.596-605; fcn ASC-093, 3 juvs, intertidal pools, Shelly Beach, 16 April 2008, NHMUK 2014.606-608.

\section{Family PANOPEIDAE Ortmann, 1893 \\ Panopeus hartii Smith, 1869}

Manning \& Chace, 1990, p. 57, figures 37, 38.

\section{MATERIAL EXAMINED}

fcn ASC-016, 1 O , cw 6.9, intertidal, rocky beach west of North East Bay, 11 April 2008, NHMUK 2014.772; fcn ASC-039, $2 \sigma^{7}$, cw 2.8, 3.0, 1 ov. O+, $\mathrm{cw} 4.0$, inside maerl balls, $2 \mathrm{~m}$, Comfortless Cove, 13 April 2008, NHMUK 2014.773-775; fon ASC-040, 1 ㅇ, cW 2.9, inside maerl balls, $2 \mathrm{~m}$, Comfortless Cove, 13 April 2008, NHMUK 2014.776; fcn ASC-069, $2 \sigma^{\top}, \mathrm{cw}$ 6.0, 8.0, 2 , cw 6.1, 8.1, intertidal, in rock pools, English Bay, North Point, 14 April 2008, NHMUK 2014.777-780; fcn ASC-083, 10', cw 4.2, intertidal, by blowhole, Collyer Point, 15 April 2008, NHMUK 2014.781; fcn ASC-095, 19, cw 10.8, intertidal pools, Shelly Beach, 16 April 2008, NHMUK 2014.781; fcn ASC-154, $20^{7}$, cw 8.9, 10.1, in rock pools, intertidal, English Bay, North Point, 17 April 2008, NHMUK 2014.783-784; fcn C6/016, $10^{7}$, cw 5.0, $9 \mathrm{~m}$, Darby wreck, 21 August 2012, NHMUK 2014.845; fcn C19/005, $10^{7}$, cw 6.5, 9.8 m, George Town Harbour, 27 August 2012, not registered; fon $\mathrm{C}_{3} 1 / 004,10^{7}, \mathrm{cW} \mathrm{4.3,1}$, CW 5.1, 12.8, Northeast Bay, 31 August 2012, NHMUK 2014.845; fcn C45/o21, 1 ov. , cw 5.0, 12.4 m, Wigan Pier, 5 September 2012, NHMUK 2014.848.

\section{Family PERCNIDAE Števčić, 2005}

Percnon abbreviatum (Dana, 1851)

Manning \& Chace, 1990, p. 68, Fig. 44.

\section{MATERIAL EXAMINED}

fcn ASC-024, 1 juv., intertidal, from rocks, Turtle Beach Point, 11 April 2008, NHMUK 2014.783-784.

\section{Percnon gibbesii (H. Milne Edwards, 1853)}

Manning \& Chace, 1990, p. 68, Fig. 45.

\section{MATERIAL EXAMINED}

fcn ASC-144, $10^{7}$, cw 15.6, diving, under Diadema, 15 m, west side of English Bay, 16 April 2008, NHMUK 2014.786; fcn
C9/oo2, 10', cw 19.8, 4.6 m, Darby Beach, 22 August 2012, NHMUK 2014.849; fcn C18/oo6, $1 \sigma^{7}$, cw 15.1, 8.7 m, Darby Beach, 25 August 2012, not registered.

Family PORTUNIDAE Rafinesque, 1815

Laleonectes vocans (A. Milne-Edwards, 1878)

Manning \& Chace, 1990, p. 50, figures 32, 33.

\section{MATERIAL EXAMINED}

fcn C36/005, 1 ㅇ, cw 20.9, 13.4 m, Wigan Pier, 2 September 2012, not registered; fcn $\mathrm{C} 47 / 002,1$ ov. 9 , $\mathrm{cW} 28.0,8 \mathrm{~m}$, Darby Wreck, 9 June 2012, NHMUK 2014.850.

Portunus (Portunus) anceps (Saussure, 1858)

Manning \& Chace, 1990, p. 52, Fig. 34.

\section{MATERIAL EXAMINED}

fcn ASC-161, 10', cW 10.9, diving, $10 \mathrm{~m}$, west side of English Bay, 17 April 2008, NHMUK 2014.787; fcn Q49/oo1, 1 , cw 24.9, $8 \mathrm{~m}$, Wigan Pier, 4 September 2012, NHMUK 2014.851.

\section{Family PSEUDOZIIDAE Alcock, 1898}

Euryozius sanguineus (Linnaeus, 1767) (Figure 2D)

Manning \& Chace, 1990, p. 55, figure 36.

\section{MATERIAL EXAMINED}

fcn ASC-132, $10^{7}, \mathrm{cW}$ 10.9, by hand, west side of English Bay, 10 m, 16 April 2008, NHMUK 2014.787; fcn 130405 Q5/B/o1, 1 ㅇ moult, cW 40.0, $16 \mathrm{~m}$, Pyramid, 21 August 2012, NHMUK 2014.852; fcn Q17/o01, 1O', cW 18.9, 8.3 m, Long Beach, 24 August 2012, ZRC 2014.0342; fcn $\mathrm{C}_{3} 8 / 003,10^{7}$, cw 12.3, 13.8 m, Pam Am Bay, 3 September 2012, NHMUK 2014. 854; fcn $\mathrm{C}_{40} / 003,1 \mathrm{O}^{7}$ moult, cw 23.9, $9.3 \mathrm{~m}$, Comfortless Cove, 4 September 2012, NHMUK 2014.855; fcn C47/o01, 1 ov. + , cw 35.5, $8 \mathrm{~m}$, Darby Wreck, 6 September 2012, NHMUK 2014.856.

Family XANTHIDAE MacLeay, 1838

Cataleptodius olsoni Manning \& Chace, 1990

Manning \& Chace, 1990, p. 54, figure 35.

\section{MATERIAL EXAMINED}

fcn ASC-018, 1 $9, \mathrm{cw} 4.6,1$ ov. + , cw 8.0, hand collecting, intertidal, from Sabellaria, Turtle Beach Point, 11 April 2008, NHMUK 2014.789-790; fcn ASC-019, 50', cw 3.6$6.9,3$, cW 4.9-7.3, hand collecting, intertidal, between oysters, Turtle Beach Point, 11 April 2008, NHMUK 2014.791-798; fon ASC-021, 4O', 3.6-5.3, 3우 cw 3.8-4.3, hand collecting, intertidal, from algal clumps, Turtle Beach Point, 11 April 2008, NHMUK 2014.799-805; fcn ASC-024, 1 ㅇ ov., cw 5.9, hand collecting, intertidal, from rocks, Turtle Beach Point, 11 April 2008, NHMUK 2014.806; fcn ASC-031, 1 \&, $\mathrm{CW} \mathrm{3.8,} \mathrm{hand} \mathrm{collecting,} \mathrm{intertidal,} \mathrm{from} \mathrm{algal}$ clumps, McArthur Point, 12 April 2008, NHMUK 2014.807; fcn ASC-035, 1 ov. , $\mathrm{cw}$ 7.2, hand collecting, intertidal, from algal clumps, McArthur Point, 12 April 2008, NHMUK 2014.808; fcn ASC-126, $10^{7}$, cw 4.4, 1 , cw 4.5, 1 ov. + , 5.4, from coralline algae, intertidal pools, east of 
McArthur Point/Turtle Beach, 17 April 2008, NHMUK 2014.809-811; fcn ASC-127, 307, cw 3.4-5.3, 19, cw 3.8, from coralline algae, intertidal pools, east of McArthur Point/Turtle Beach, 17 April 2008, NHMUK 2014.812-815; $\mathrm{fcn} \mathrm{A} 2 / 001,1$, $\mathrm{cW} 4.8$, no further details, not registered.

\section{Microcassiope minor (Dana, 1852)}

Manning \& Chace, 1990, p. 56.

\section{MATERIAL EXAMINED}

fcn C8/003, 10', cw 5.6, Darby Beach, 22 August 2012, NHMUK 2014.857; fon C10/004, $10^{7}$, cw 5.3, 1 , cw 5.8, Red Rock, 23 August 2012, NHMUK 2014.858-859; fcn $\mathrm{C}_{35} / 003,10^{7}, \mathrm{cw} 4.6,8.5 \mathrm{~m}$, Northeast Bay, 31 August 2012, NHMUK 2014.860; fcn Q7/002, 19, cw 5.3, $16.8 \mathrm{~m}$, Two Hooks, 22 August 2012, NHMUK 2014.861; 107, cW 5, 1 ov. , $\mathrm{cw} 7.9,2012$ no further details, not registered.

Nanocassiope melanodactylus (A. Milne Edwards, 1868)

Manning \& Chace, 1990, p. 57 (as Nannocassiope melanodactylus, erroneous spelling).

MATERIAL EXAMINED

fcn C45/018,10', cw 9.6, 12.4 m, Wigan Pier, 5 September 2012, not registered.

\section{Paractaea rufopunctata africana Guinot, 1976}

Manning \& Chace, 1990, p. 57, Fig. 39.

\section{MATERIAL EXAMINED}

fcn Co9/001, 1 $O$, cw 13.1, 4.6 m, Darby Beach, 22 August 2012, NHMUK 2014.862; fcn C18 005, 107, cw 16.1, 8.7 m, Darby Beach, 25 August 2012, NHMUK 2014.863.

Platypodiella picta (A. Milne Edwards, 1899)

Manning \& Chace, 1990, p. 59.

\section{MATERIAL EXAMINED}

fcn IT18/o02, 1 ov. $ᄋ$, cw 16.3, west of English Bay, intertidal, 2 September 2012, NHMUK 2014.864; fon C33/oo1, 1 ov. O , cw 8.3, Boatswain Bird Island, 16 m, 1 September 2012, not registered.

\section{Williamstimpsonia denticulatus (White, 1848)}

Manning \& Chace, 1990, p. 60, figure 40 (as Xanthodius denticulatus).

\section{MATERIAL EXAMINED}

$\mathrm{fcn}$ ASC-001, 19, cw 11.0, from Sabellaria Reef, $1 \mathrm{~m}$, Comfortless Cove, 10 April 2008, NHMUK 2014.816; fcn ASC-002, 3O', cw 5.8-6.4, from Sabellaria Reef, $1 \mathrm{~m}$, Comfortless Cove, 10 April 2008, NHMUK 2014.817-819; fcn ASC-032, 2 juvs, intertidal, from algal clumps, McArthur Point, 12 April 2008, NHMUK 2014.820-821; fcn ASC-039, 3 juvs, inside maerl balls, $2 \mathrm{~m}$, Comfortless Cove, 13 April 2008, NHMUK 2014.822-824; fcn ASC-061, 2우, $\mathrm{cw} 6.0,6.9,3 \sigma^{7}, \mathrm{cW} 8.0-10.8$, in coralline algal concretions, $2 \mathrm{~m}$, Comfortless Cove, 13 April 2008, NHMUK 2014.825-829; fcn ASC-076, 1 cw 8.4, 1 juv., from maerl ball, $5 \mathrm{~m}$, Comfortless Cove, 14 April 2008, NHMUK 2014.830-831; fcn ASC-079, 1 으, cw 14.0, intertidal, by blowhole, Collyer Point, 15 April 2008, NHMUK 2014.830-831; fcn ASC-083, 107, cw 7.4, 1 juv. intertidal, by blowhole, Collyer Point, 15 April 2008, NHMUK 2014.833; fcn ASC-138, 1 으, cw 11.7, 10 m, West side of English Bay, 16 April 2008, NHMUK 2014.834; fon ASC-141, 1 , cw 14.4, in rock pools, intertidal, English Bay, North Point, 16 April 2008, NHMUK 2014.835; fcn ASC-145, $10^{7}, \mathrm{cw} 5.2,15 \mathrm{~m}$, west side of English Bay, 17 April 2008, NHMUK 2014.836; fcn ASC-157, 19, cw 15.8, in rock pools, intertidal, English Bay, North Point, 17 April 2008, NHMUK 2014.837; fcn C6/o16, 20', cW 18.2, 18.2, 9 m, Darby Wreck, 21 August 2012, NHMUK 2014.865-866, NHMUK 2014.837; fcn C8/ 002,1 ㅇ, cw 10.3, Darby Beach, 22 August 2012, NHMUK 2014.867; fcn C10/o03, 107, cw 13.7, Red Rock, 23 August 2012, NHMUK 2014.868; fcn C10/o04, 1 , cw 17.1, Red Rock, 23 August 2012, NHMUK 2014.869; fcn C20/007, $10^{7}, \quad \mathrm{cW} 15.5,11 \mathrm{~m}$, Sudan Wreck, 27 August 2012, NHMUK 2014.870; fcn $\mathrm{C}_{31} / 008,10^{7}$, cw. 15.9, diving, $12.8 \mathrm{~m}$, North East Bay, 31 August 2012, NHMUK 2014.871; fcn C45/o22, 1 , cw 10.0, 12.4 m, Wigan Pier, 5 September 2012, NHMUK 2014.872; fcn C50/006, $10^{7}, \mathrm{cW}$ 19.3, $12 \mathrm{~m}$, Wigan Pier, 7 September 2012, NHMUK 2014.873 .

\section{DISCUSSIDN}

Manning \& Chace (1990) recorded 74 species of Decapoda from Ascension Island, including the terrestrial crab, Johngarthia lagostoma, and the anchialine shrimps, Procaris ascensionis and Typhlatya rogersi. All three of these species were also noted during the 2008 and 2012 surveys, but are herein not further reported upon. Since this study there have been few amendments to the fauna. The western Atlantic shrimp, Lysmata intermedia was recorded from Ascension by Manning \& Chace (1990), however Manning (in litt.) in d'Udekem d'Acoz (2000) indicates this to be a misidentification, with the specimens probably belonging to the east Atlantic L. seticaudata (see Table 2). Manning \& Chace (1990) also discuss the presence of Latreillia manningi at Ascension, this species was, however, synonymized with L. elegans by Castro et al. (2003) and is reported herein under that name. Fransen (2006) in a partial key to Periclimenaeus, states Periclimenaeus atlanticus to be present on the island, however this is considered a lapsus (Fransen, in litt.). In addition to Corallianassa hartmeyeri reported by Manning \& Chace (1990), C. longiventris is herein also recorded for Ascension Island. Only two species of shrimp have been added to the known fauna of the island since Manning \& Chace (1990). De Grave (2010) described Typton holthuisi from Comfortless Cove, noting that this species was potentially more widely distributed in the tropical eastern Atlantic. Anker \& De Grave (2012) described Alpheus cedrici from English Bay, a species still only known from Ascension. In the present contribution, a further species is added, the widespread Gnathophyllum americanum. Despite intensive collecting in 2008 and 2012, no additional crab species are reported herein.

The presently known marine Decapoda fauna thus stands at 75 species (Table 2), of which 12 species are considered to be endemic to the island. A full discussion of the 
biogeography of the marine decapod fauna is beyond the scope of the present contribution, but it is noted that the decapod fauna of St Helena is poorly known, being restricted to Chace (1966) and De Grave (2007), who note 36 species to occur. There is a likelihood that a number of the endemic taxa recorded from Ascension will eventually be reported from St Helena.

\section{ACKNDWLEDGEMENTS}

This publication partly arises from research funded by the John Fell Oxford University Press Research Fund, who funded the 2008 OUMNH expedition to Ascension Island by S. and H. De Grave. The funding for the 2012 expedition came from a grant to Dr Paul Brickle from the Darwin Initiative (EIDCFo12). We are grateful to the Shallow Marine Surveys Group and the South Atlantic Environmental Research Institute for organizing the 2012 expedition. We are also very grateful to Ascension Island Government, the members of staff at the Conservation Centre and Ascension Island Dive Club for their cooperation, accommodation and hospitality during both expeditions. Finally we are grateful to British Forces South Atlantic Islands for their logistical support to the 2012 expedition.

\section{REFERENCES}

Anker A. (2007) New species and records of alpheid shrimps, genera Salmoneus Holthuis and Parabetaeus Coutière, from the tropical western Atlantic (Decapoda, Caridea). Zootaxa 1653, 21-39.

Anker A. (2010) The shrimp genus Salmoneus Holthuis, 1955 (Crustacea, Decapoda, Alpheidae) in the tropical western Atlantic, with description of five new species. In De Grave S. and Fransen C.H.J.M. (eds), Contributions to shrimp taxonomy. Zootaxa 2372, 177-205.

Anker A. and De Grave S. (2012) Description of Alpheus cedrici sp.n., a strikingly coloured snapping shrimp (Crustacea, Decapoda, Alpheidae) from Ascension Island, central Atlantic Ocean. Zookeys $183,1-15$.

Banner D.M. and Banner A.H. (1985) The alpheid shrimp of Indonesia based upon J.G. De Man's “The Decapoda of the Siboga Expedition, Part II. Family Alpheidae." (1911). Marine Research in Indonesia $25,1-79$.

Benedict J.E. (1893) Notice on the crustaceans collected by the United States Scientific expedition to the west coast of Africa. Proceedings of the United States National Museum 16, 535-541.

Castro P., Williams A.B. and Cooper L.L. (2003) Revision of the family Latreilliidae Stimpson, 1858 (Crustacea, Decapoda, Brachyura). Zoosystema 25, 601-634.

Chace F.A. Jr (1966) Decapod crustaceans from St. Helena Island, South Atlantic. Proceedings of the United States National Museum 3536, 623-661.

Chace F.A. Jr (1972) The shrimps of the Smithsonian-Bredin Caribbean expeditions with a summary of the West Indian shallow-water species (Crustacea: Decapoda: Natantia). Smithsonian Contributions to Zoology 98, 1-179.

Chace F.A. Jr and Manning R.B. (1972) Two new caridean shrimps, one representing a new family, from marine pools on Ascension Island (Crustacea: Decapoda: Natantia). Smithsonian Contributions to Zoology 131, 1-18.
Crosnier A. and Forest J. (1966) Crustacés Décapodes: Alpheidae. Campagne de la Calypso dans le Golfe de Guinée et aux Iles Principe, São Tome et Annobon (1956), et Campagne aux Iles du Cap Vert (1959) (suite), XXVII: résultats scientifique des campagnes de la "Calypso", Fascicule 7. Annales de l'Institut Océanographique $44,199-314$.

d'Udekem d'Acoz C. (2000) Redescription of Lysmata intermedia (Kingsley, 1879) based on topotypical specimens, with remarks on Lysmata seticaudata (Risso, 1816) (Decapoda, Caridea, Hippolytidae). Crustaceana $73,719-735$.

De Grave S. (2007) On the occurrence of Gnathophylleptum tellei d'Udekem d'Acoz, 2001 (Decapoda, Gnathophyllidae) in St Helena, South Atlantic Ocean. Crustaceana 80, 893-895.

De Grave S. (2010) A new species of the genus Typton Costa (Decapoda, Palaemonidae, Pontoniinae) from Ascension Island. In Fransen C.H.J.M., De Grave S. and Ng P.K.L. (eds) Studies on Malacostraca: Lipke Bijdeley Holthuis Memorial Volume. Crustaceana Monographs, 14. Leiden: Brill, pp. 209-218.

Fransen C.H.J.M. (2006) On Pontoniinae (Crustacea, Decapoda, Palaemonidae) collected from ascidians. Zoosystema 28, 713-746.

Holthuis L.B. (1951) The caridean Crustacea of tropical West Africa. Atlantide Report. Scientific Results of the Danish Expedition to the Coasts of Tropical West Africa 1945-1946 2, 7-187.

Kropp R.K. and Manning R.B. (1987) The Atlantic gall crabs, family Cryptochiridae (Crustacea: Decapoda: Brachyura). Smithsonian Contributions to Zoology 462, 1-21.

Lenz H. and Strunck K. (1914) Die Dekapoden der Deutschen Südpolar-Expedition 1901-1903. I. Brachyuren und Macruren mit ausschluss der Sergestiden. Wissenschaftliche Ergebnisse der Deutschen Südpolar-Expedition 15(Zoologie 7), 257-346.

Linnaeus C. (1754) Specimen academician s[i]stens Chinensia Lagerstromiana quod ... praeside ... Carolo Linnaeo ... submittit J.L. Odhelio ... d. [23.] Decembr. Holmiae.

Linnaeus C. (1771) Mantissa plantarum altera, Generum editionis VI. Specierum editionis II, Volume 2. Holmiae.

Manning R.B. (1988) The status of Callianassa hartmeyeri Schmitt, 1935 with the description of Corallianassa xutha from the west coast of America (Crustacea, Decapoda, Thalassinidea). Proceedings of the Biological Society of Washington 101, 883-889.

Manning R.B. and Chace F.A. Jr (1990) Decapod and stomatopod Crustacea from Ascension Island, South Atlantic Ocean. Smithsonian Contributions to Zoology 503, 1-91.

Miers E.J. (1881) On a collection of Crustacea made by Baron Hermann-Maltzam at Goree Island, Senegambia. The Annals and Magazine of Natural History 8, 204-220, 259-281, 364-377, Plates $13-16$.

Nomura K. and Anker A. (2001) A redescription of Parabetaeus culliereti Coutière and status of the genus Neoalpheopsis Banner (Decapoda: Caridea: Alpheidae). Crustacean Research 30, 43-54.

Ortmann A. (1893) Decapoden und Schizopoden. Ergebnisse der Plankton-Expedition der Humboldt-Stiftung 2, 1-120.

Rathbun M.J. (1930) The Cancroid crabs of America. United States National Museum Bulletin 152, 1-609.

Sakai K. (1999) Synopsis of the family Callianassidae, with keys to subfamilies, genera and species, and the description of new taxa (Crustacea: Decapoda: Thalassinidea). Zoologische Verhandelingen $326,1-152$.

Sakai K. (2005) Callianassoidea of the world (Decapoda, Thalassinidea). Crustaceana Monographs 4, 1-200. 
Sakai K. (2011) Axioidea of the world and a reconsideration of the Callianassoidea (Decapoda, Thalassinidea, Callianassida). Crustaceana Monographs 13, 1-616.

Schmitt W.L. (1935) Mud shrimps of the Atlantic coast of North America Smithsonian Miscellaneous Collections 93, 1-21.

Stebbing T.R.R. (1914) Stalk-eyed Crustacea Malacostraca of the Scottish National Antarctic Expedition. Transactions of the Royal Society of Edinburgh 50, 253-307.

Studer T. (1883) Verzeichniss der während der Reise S.M.S. Gazelle an der Westküste von Afrika, Ascension und dem Cap der guten Hoffnung gesammelten Crustaceen. Abhandlungen der K. Preussischen Akademie der Wissenschaften, Berlin 2, 1-32.
White A. (1847) List of the specimens of Crustacea in the collection of the British Museum. London: British Museum.

and

Wicksten M.K. and McClure M.R. (2003) A new species of Alpheus (Decapoda: Caridea: Alpheidae) from the Gulf of Mexico. Crustacean Research 32, 26-31.

\section{Correspondence should be addressed to:}

S. De Grave

Oxford University Museum of Natural History, Oxford, UK email: sammy.degrave@oum.ox.ac.uk 